\title{
A Hole Theory of Polymer Liquids and Glasses. V. Surface Tension of Polymer Liquids
}

\author{
Takuhei Nose \\ Department of Polymer Technology, Tokyo Institute of \\ Technology, Tokyo, Japan.
}

(Received July 24, 1970)

\begin{abstract}
A simple theory of surface tension $\gamma$ for polymer liquids is presented with the use of a hole theory of polymer liquids. It is found that if $\gamma\left(\sigma^{3} / c\right)^{2 / 3} c / \varepsilon^{*} z^{\prime}$ is taken as the reduced surface tension the principle of corresponding states is in satisfactory accord with theory, where $\sigma$ is a characteristic parameter for separation involved in the Lennard-Jones $12-6$ potential; $\varepsilon^{*}$ is that for potential energy; $3 c$ is the number of external degrees of freedom per segment, and $z^{\prime}$ is the average coordination number of a segment. The theory makes it possible to interpret the so-called Hildebrand equation for the relation between $r$ and cohesive energy density, and shows that the parachor is proportional to the product of the 13/12-th power of an occupied volume $\sigma^{3} \mathrm{~N}$ and the 1/4-th power of a characteristic cohesive energy density, $0.699 \varepsilon^{*} z^{\prime} / \sigma^{3}$, when an appropriate atomic group is taken.
\end{abstract}

KEY WORDS Hole Theory / Polymer Liquids / Surface Tension / Corresponding States / Cohesive Energy Density / Parachor /

The surface tension is a fundamental thermodynamic quantity of liquids reflecting intermolecular interaction, and as for polymer liquids it has been recently investigated by Schonhorn, et al., ${ }^{1}$ Dettre and Johnson, ${ }^{2}$ Roe, ${ }^{3}$ and by Hata, et al $^{4}{ }^{4} \mathrm{Roe}^{3}$ has applied Prigogine's hole theory ${ }^{5}$ of simple liquids to molten polymers and compared the theoretical results with experimental ones using the molecular parameters obtained by Simha, et al. ${ }^{6}$ Here we present a simple theory for the surface tension of polymer liquids using the hole theory proposed in a previous paper $^{7}$ and derive the relation between surface tension and cohesive energy density. Furthermore we express the "parachor" by the molecular parameters involved in the present theory to clarify its physical meaning.

\section{THEORY}

First we rewrite the partition function for polymer liquids previously obtained. ${ }^{7}$ Considering the system in which the $N / r$ polymer molecules, each consisting of $r$ segments, are distributed on a lattice comprising $M$ sites $(M \geq N)$, we may write the partition function $Z$ for the system as
$Z=J(T) \sum_{\lambda} g_{\lambda} q^{c N} \bar{v}_{\mathrm{f}}{ }^{c N} \exp \left\{-\varphi(0)\left(z^{\prime} N-n_{01}^{*}\right) / 2 k T\right\}$

where $J(T)$ is the partition function associated with intrasegmental degrees of freedom as a function of temperature $T ; 3 c N$ is the total number of external degrees of freedom; $q^{c N}$ is a kinetic part of the partition function associated with external degrees of freedom; $z^{\prime}$ is the average coordination number of a segment, which is related to the coordination number $z$ of a lattice site by the equation $z^{\prime}=(z r-2 r+2) / r$; $z^{\prime} \varphi(\rho)$ is the mean potential energy of a segment having no neighboring hole at a point which deviates from the center of the cell by the distance $\rho ; \sum_{\lambda} g_{\lambda}$ represents the combinatory factor, arising from the mixing of molecules and holes; and $n_{01}^{*}$ is the most probable number of segmenthole pairs which is expressed by

$$
n_{\mathrm{ol}}^{*}=\frac{z^{\prime} N(M-N) z}{(M-N) z+z^{\prime} N}
$$

The symbol $\bar{v}_{\mathrm{f}}$ is the average free volume given by $\int_{\mathrm{e} e 11} \exp \left[-\left(1-\omega^{*}\right) z^{\prime}\{\varphi(\rho)-\varphi(0)\}\right] / k T \mathrm{~d} \rho$, where $\omega^{*}$ is the most probable value of $\omega$ ( $\omega$ is the 
fraction of empty sites in the nearest neighbors) By the use of the well-known approximation, ${ }^{8}$

$$
\ln v_{\mathrm{f}}=\omega \ln v_{\mathrm{f} 1}+(1-\omega) \ln v_{\mathrm{fo}}
$$

and the relation $z^{\prime} N \omega^{*}=n_{\mathrm{ol}}^{*}$, the value $\bar{v}_{\mathrm{f}}$ can be written as follows

$$
\bar{v}_{\mathrm{f}}=v_{\mathrm{f} 1}^{\omega *} v_{\mathrm{fo}}^{1-\omega *}=v_{\mathrm{fo}}\left(v_{\mathrm{f} 1} / v_{\mathrm{fo}}\right)^{n_{\mathrm{O} 1}^{*} / N z^{\prime}}
$$

where $v_{\mathrm{f} 1}$ and $v_{\text {fo }}$ are usually taken to be the free volume, $v_{\mathrm{f}}$, at $\omega=1$ and $v_{\mathrm{f}}$ at $\omega=0$ respectively. However they are considered to be arbitary parameters determined so that the value of $v_{\mathrm{f}}$ is approximated by eq 3 in the range of $v_{\mathrm{f}}$ considered. We now derive surface tension $\gamma$ in the conventional manner:

$$
\gamma=-k T(\partial \ln Z / \partial \Omega)_{T, V, N_{\mathrm{B}}+N_{\mathrm{S}}}
$$

where $\Omega$ denotes the surface area; $V$ is the volume; and the subscripts $B$ and $S$ refer to bulk and surface phases respectively.

In the present lattice model for surface, we suppose that the segment density varies discontinuously from the constant value of the bulk density to zero at the surface, the vapor phase being disregarded. Then, since there is no segment outside the first lattice layer at the surface, the number of segments occupying the nearest neighbor sites in the first layer is different from those in the bulk, though the segment density or the hole fraction is assumed to be the same. Accordingly, the free volume and the lattice energy of the first layer may be different from that of the bulk phase. Those of the second, third, and more interior layers may, however, be approximately equal to each other. Hence, only the first lattice layer is here regarded as the surface phase having different free volume and different lattice energy from those of the bulk phase. It is also assumed that the hole fraction in the surface layer is identical with that in bulk, i.e., $N_{\mathrm{S}} / M_{\mathrm{S}}=N_{\mathrm{B}} / M_{\mathrm{B}}$, and that the configuration of chain molecules at the surface is no difference from that in bulk, namely the term $J(T) \sum_{\lambda} g_{\lambda}$ is independent of surface area.

In the case of the system including the surface phase, the partition function $Z$ of eq 1 is transformed into

$$
\begin{aligned}
Z= & J(T) \sum_{\lambda} g_{\lambda} q^{c\left(N_{\mathrm{B}}+N_{\mathrm{S}}\right)}{ }_{\mathrm{B}} \bar{v}_{\mathrm{f}}{ }^{c N_{\mathrm{B}}} \overline{\mathrm{s}}_{\mathrm{f}}{ }^{c N_{\mathrm{S}}} \\
& \times \exp \left[-\varphi(0)\left\{z^{\prime}\left(N_{\mathrm{B}}+N_{\mathrm{S}}\right)-\left({ }_{\mathrm{B}} n_{\mathrm{o} 1}^{*}+{ }_{\mathrm{s}} n_{\mathrm{o} 1}^{*}\right)\right\} / 2 k T\right]
\end{aligned}
$$

Thus, the problem is reduced to the evaluation of ${ }_{\mathrm{s}} n_{01}^{*}$ and ${ }_{\mathrm{s}} \bar{v}_{\mathrm{f}}$, since ${ }_{\mathrm{B}} n_{\mathrm{O} 1}^{*}$ and ${ }_{\mathrm{B}} \bar{v}_{\mathrm{f}}$ are given by eq 2 and 4 .

Let $m$ be the ratio of the number of the nearest neighbor sites in the surface layer to that in the interior (for example, $m=9 / 12$ for hexagonal packing). Then, considering the assumption $M_{\mathrm{S}} / N_{\mathrm{S}}=M_{\mathrm{B}} / N_{\mathrm{B}}=M v^{*} / N v^{*} \equiv V / V^{*} \equiv \tilde{V}$, where $v^{*}$ is the cell volume, we can express ${ }_{\mathrm{B}} n_{\mathrm{O} 1}^{*}$ and ${ }_{\mathrm{S}} n_{\mathrm{ol}}^{*}$, by the use of eq 2 , as

$$
\begin{gathered}
{ }_{\mathrm{B}} n_{\mathrm{ol}}^{*}=z^{\prime} \phi N_{\mathrm{B}} \\
{ }_{\mathrm{S}} n_{01}^{*}=z^{\prime} \phi N_{\mathrm{S}} m+z^{\prime}(1-m) N_{\mathrm{S}} \\
=\frac{\left(M_{\mathrm{B}}-N_{\mathrm{B}}\right) z}{\left(M_{\mathrm{B}}-N_{\mathrm{B}}\right) z+z^{\prime} N_{\mathrm{B}}}=\frac{\left(M_{\mathrm{S}}-N_{\mathrm{S}}\right) z}{\left(M_{\mathrm{S}}-N_{\mathrm{S}}\right) z+z^{\prime} N_{\mathrm{S}}} \\
=(\tilde{V}-1) /(\tilde{V}-1+s)
\end{gathered}
$$

Here we put $s=z^{\prime} \mid z$.

If the value of ${ }_{\mathrm{s}} \bar{v}_{\mathrm{f}}$ is expressed in the form of eq 3 , we have

$$
\ln _{\mathrm{s}} \bar{v}_{\mathrm{f}}=\omega_{\mathrm{S}}^{*} \ln _{\mathrm{s}} v_{\mathrm{f} 1}+\left(1-\omega_{\mathrm{S}}^{*}\right) \ln _{\mathrm{s}} v_{\mathrm{fo}}
$$

where $\omega_{\mathrm{S}}{ }^{*}$ is the most probable fraction of the number of holes in the nearest neighbor sites $z^{\prime} m$ in the surface layer and is identical with that in bulk according to the assumption $M_{\mathrm{S}} / N_{\mathrm{S}}=M_{\mathrm{B}} / N_{\mathrm{S}}$, i.e., $\omega_{\mathrm{S}}{ }^{*}={ }_{\mathrm{B}} n_{\mathrm{o} 1}^{*} / z^{\prime} N_{\mathrm{B}}=\phi$ from eq 6. Accordingly we have

$$
{ }_{\mathrm{s}} \bar{v}_{\mathrm{f}}={ }_{\mathrm{s}} v_{\mathrm{fo}}\left({ }_{\mathrm{s}} v_{\mathrm{f} 1} / \mathrm{s} v_{\mathrm{fo}}\right)^{\phi}
$$

and

$$
{ }_{\mathrm{B}} \bar{v}_{\mathrm{f}}={ }_{\mathrm{B}} v_{\mathrm{fo}}\left({ }_{\mathrm{B}} v_{\mathrm{f} 1} /{ }_{\mathrm{B}} v_{\mathrm{fo}}\right)^{\phi}
$$

Finally, from eq $1^{\prime}$ with eq $6,7,10$, and 11 , we obtain the partition function as

$$
\begin{aligned}
Z= & J(T) \sum_{\lambda} g_{\lambda} q^{c\left(N_{\mathrm{B}}+N_{\mathrm{S}}\right)} \\
& \times \exp \left\{-z^{\prime} \varphi(0)\left(N_{\mathrm{B}}+N_{\mathrm{S}}\right) / 2 k T\right\} \\
& \times{ }_{\mathrm{B}} v_{\mathrm{fo}}^{c(1-\phi) N_{\mathrm{B}}} v_{\mathrm{fo}}^{c(1-\rho) N_{\mathrm{S}_{\mathrm{B}}} v_{\mathrm{f} 1}^{c \phi N_{\mathrm{B}}} v_{\mathrm{f} 1}^{c \phi N}} \\
& \times \exp \left[\varphi(0) z^{\prime}\left\{\phi\left(N_{\mathrm{B}}+N_{\mathrm{S}} m\right)+N_{\mathrm{S}}(1-m)\right\} / 2 k T\right]
\end{aligned}
$$

To calculate surface tension from eq 5 , we need to express surface area $\Omega$ in terms of $M_{\mathrm{S}}$ or $N_{\mathrm{S}}$. It may be neither possible nor logical, however, to treat this problem strictly, on the 
A Hole Theory of Polymer Liquids and Glasses. V.

basis of the present theory because of the simplicity of the model. Therefore, in estimating surface area, it is here assumed that a segment in the surface layer can be replaced by a sphere having a cell volume $v^{*} / c$ which is the cell size of a segment having three external degrees of freedom. (The size of a segment can be arbitrarily chosen except for such problems as evaluation of surface area or those associated with the number of segments). This evaluation is equivalent to that of Roe. ${ }^{3}$ We have

$$
\Omega=\eta\left(v^{*} / c\right)^{2 / 3} c M_{\mathrm{S}}
$$

or

$$
\Omega=\eta\left(v^{*} / c\right)^{2 / 3} c N_{\mathrm{S}} \tilde{V}
$$

where $\eta$ is a shape factor and for hexagonal packing $\eta=\left(3^{1 / 2} / 2\right) 2^{1 / 3}=1.091$ because $d^{3} / 2^{1 / 2}=$ $v^{*} / c$ and $\Omega=\left(3^{1 / 2} / 2\right) d^{2} c M_{\mathrm{S}}$ where $d$ is the diameter of an equivalent sphere.

We can now derive the surface tension from eq 5,12 , and 13 , using the above mentioned two assumptions, i.e., $M_{\mathrm{S}} / N_{\mathrm{S}}=M_{\mathrm{B}} / N_{\mathrm{B}}=\tilde{V}$ and $J(T) \sum_{\lambda} g_{\lambda}$ is independent of surface area $\Omega$. The result is

$$
\begin{aligned}
\gamma= & \varepsilon^{*} z^{\prime}\left\{\eta\left(v^{*} / c\right)^{2 / 3} c\right\}^{-1} \\
& \times s \tilde{V} \tilde{V}^{-1}(\tilde{V}-1+s)^{-1}\{K(1-m)-f \widetilde{T}\}
\end{aligned}
$$

where

$$
\begin{aligned}
f & =\ln \left({ }_{\mathrm{s}} v_{\mathrm{fo}} /{ }_{\mathrm{B}} v_{\mathrm{fo}}\right)+s(\tilde{V}-1) \ln \left({ }_{\mathrm{s}} v_{\mathrm{f} 1} /{ }_{\mathrm{B}} v_{\mathrm{f} 1}\right) \\
K & =-\varphi(0) / 2 \varepsilon^{*} \\
& =\left\{2.409\left(\sigma^{3} N / V^{*}\right)^{2}-1.0109\left(\sigma^{3} N / V^{*}\right)^{4}\right\} / 2
\end{aligned}
$$

and

$$
\tilde{T} \equiv T / T^{*} \equiv c k T / \varepsilon^{*} z^{\prime}
$$

The symbols $\varepsilon^{*}$ and $\sigma$ are the parameters involved in the Lennard-Jones 12-6 potential expressed as $u\left(r_{i j}\right)=4 \varepsilon^{*}\left\{\left(\sigma / r_{i j}\right)^{12}-\left(\sigma / r_{i j}\right)^{6}\right\}$, where $u\left(r_{i j}\right)$ is the potential energy of interaction between segments $i$ and $j$ as a function of separation $r_{i j}$. The derivation of $K$ was given in the previous paper. ${ }^{7}$

We must here estimate the value of $f$. The second term in eq 15 is not significant and will vanish when we take ${ }_{\mathrm{s}} v_{\mathrm{f} 1}={ }_{\mathrm{B}} v_{\mathrm{f} 1}=v_{\mathrm{f}}(\omega=1)=v^{*}$, which is not always necessary, but reasonable and convenient. We consider the case in which ${ }_{\mathrm{s}} v_{\mathrm{f} 1}={ }_{\mathrm{B}} v_{\mathrm{f} 1},{ }_{\mathrm{s}} v_{\mathrm{fo}}={ }_{\mathrm{s}} v_{\mathrm{f}}(\omega=0)$ and ${ }_{\mathrm{B}} v_{\mathrm{fo}}={ }_{\mathrm{B}} v_{\mathrm{f}}(\omega=0)$ and will estimate these quantities with the square well potential which was used previously ${ }^{7}$ for the estimation of the free volume.

By analogy with the expressions in the hole theory for surface tension presented by Prigogine and Saraga, ${ }^{5}$ we have

$$
\begin{aligned}
\mathrm{s}_{\mathrm{f}} v_{\mathrm{f}}(\omega=0)= & (4 \pi / 3)(a-\sigma)^{3} / 2 \\
& +(4 \pi / 3)(a-\sigma)^{2}\{a-\sigma(1-\beta)\} / 2 \\
{ }_{\mathrm{B}} v_{\mathrm{f}}(\omega=0)= & (4 \pi / 3)(a-\sigma)^{3}
\end{aligned}
$$

which was also used by Ree, et al., ${ }^{9}$ where $\sigma$ is the parameter involved in the L-J $12-6$ potential; $a$ is the distance between the nearest neighbor sites, and $a-\sigma(1-\beta)$ is the width of square well potential for the perpendicular motion towards the outside at surface. Then, putting $a^{3} / 2^{1 / 2}=v^{*}$ for hexagonal packing, we derive

$$
\begin{aligned}
f= & \ln \left({ }_{\mathrm{s}} v_{\mathrm{fo}} / \mathrm{s} v_{\mathrm{fo}}\right)=\ln { }_{\mathrm{s}} v_{\mathrm{f}}(\omega=0) /{ }_{\mathrm{B}} v_{\mathrm{f}}(\omega=0) \\
= & \ln \left[1 / 2+(1 / 2)\left\{1-(1-\beta) 2^{-1 / 6}\left(\sigma^{3} N / V^{*}\right)^{1 / 3}\right\}\right. \\
& \left.\times\left\{1-2^{-1 / 6}\left(\sigma^{3} N / V^{*}\right)^{1 / 3}\right\}^{-1}\right]
\end{aligned}
$$

We can now calculate surface tension using eq 14 for a given value of $\beta$, using the equation of state for the cell volume obtained in the previous paper, ${ }^{7}$ since $K$ and $f$ are expressed as a function of $V^{*} / \sigma^{3} N$. On the other hand, if we consider a model treated in the previous paper, ${ }^{7}$ where the volume of the cell is $\sigma^{3}$ and unchanged with temperature, eq 14 becomes a simpler form, because $K$ and $f$ can be put as constants. In this case, for hexagonal packing, $\eta=1.091, m=9 / 12$ as mentioned already, and $K=0.699$ for $V^{*}=\sigma^{3} N$ from eq 16 . eq 14 then becomes

$$
\begin{aligned}
\tilde{\gamma} & =\gamma\left(\sigma^{3} / c\right)^{2 / 3} c / \varepsilon^{*} z^{\prime} \\
& =s \tilde{V}^{-1}(\tilde{V}-1+s)^{-1}\left(0.160_{1}-0.917 f \tilde{T}\right)
\end{aligned}
$$

where $\tilde{V}=V / \sigma^{3} N$, and the value of $f$ is constant.

The temperature dependence of surface tension at constant pressure is derived from eq 21 for the constant cell volume model as

$$
\begin{aligned}
- & (\partial \ln \gamma / \partial T)_{p} / \alpha \\
= & \left\{2-(1-s) \tilde{V}^{-1}\right\}\left\{1-(1-s) \tilde{V}^{-1}\right\}^{-1} \\
& +(\partial \ln \tilde{V} / \partial \ln \tilde{T})_{p}{ }^{-1}\left\{0.174_{8} /(f \tilde{T})-1\right\}^{-1}
\end{aligned}
$$

or

$$
\begin{aligned}
- & (\partial \ln \gamma / \partial \ln T)_{p} \\
= & (\partial \ln \tilde{V} / \ln \tilde{T})_{p}\left\{2-(1-s) \tilde{V}^{-1}\right\}\left\{1-(1-s) \tilde{V}^{-1}\right\}^{-1} \\
\quad & +\left\{0.174_{8} /(f \tilde{T})-1\right\}^{-1}
\end{aligned}
$$


where $\alpha=(\partial V / \partial T)_{p} / V$, thermal expansion coefficient.

On the other hand, the relation between surface tension and cohesive energy density can be derived as follows. According to the present hole theory, cohesive energy density $D$ can be expressed by the equation

$$
D=K \varepsilon^{*} N z^{\prime} \tilde{V}^{-1} s(\tilde{V}-1+s)^{-1} / V^{*}
$$

as given in the previous paper. ${ }^{7}$ It then follows from eq 21 and 24 with $K=0.699$ and $V^{*}=\sigma^{3} N$ that

$$
\left(\gamma / V^{1 / 3}\right) / D=0.496 c^{-1 / 3} \tilde{V}^{-1 / 3}\left(0.229-1.31_{2} f \tilde{T}\right)
$$

where $\gamma$ is expressed in dyn/cm and $D$ in cal/cc.

Expressing $c$ in terms of $\tilde{V}, \tilde{T}, T$, and $D$, we have

$$
c=V^{*} D s \tilde{T} \tilde{V}(\tilde{V}-1+s) /(0.699 R T)
$$

where $R$ denotes the gas constant. Then, combination of eq 25 and 26 yields

$$
\begin{aligned}
r /\left(D^{2} T\right)^{1 / 3}= & 0.496(0.699 R s)^{1 / 3}\{\tilde{T} \tilde{V}(\tilde{V}-1+s)\}^{-1 / 3} \\
& \times\left(0.229-1.31_{2} f \tilde{T}\right)
\end{aligned}
$$

\section{COMPARISON WITH EXPERIMENTAL OBSERVATIONS AND DISCUSSION}

\section{Surface Tension}

First we must obtain a value for $\beta$ in eq 20 , which may fall between zero and unity, in order to evaluate $f$. If we take $\beta=0.25$ as Roe, et al.,$^{3,5,9}$ did, the value of $f$ is equal to 0.704 when the cell volume $V^{*}$ is $\sigma^{3} N$. On the other hand if the value of ${ }_{s} v_{\text {fo }}$ can be approximated to the value of ${ }_{\mathrm{B}} v_{\mathrm{f}}(\omega=1-m)$ obtained by eq 3 , i.e., $\ln _{\mathrm{s}} v_{\mathrm{fo}}=(1-m) \ln { }_{\mathrm{B}} v^{\mathrm{f} 1}+m \ln _{\mathrm{B}} v_{\mathrm{fo}}$, the value of $f$ is given by $(1-m) \ln \left({ }_{\mathrm{B}} v_{\mathrm{f} 1} /{ }_{\mathrm{B}} v_{\mathrm{fo}}\right)=(1-m) \ln$ $v^{*} /{ }_{\mathrm{B}} v_{\mathrm{f}}(\omega=0)$, to become $1.21_{7}$ when $m=9 / 12$ and $V^{*}=\sigma^{3} N$. (Putting $\beta=0.5$ in eq 20 with $V^{*}=\sigma^{3} N$, we have the value of $f, 1.111$, which is very close to the value $1.21_{7}$ ). Because of the influence of the segments in the outside of nearest neighbor holes, however, ${ }_{\mathrm{B}} v_{\mathrm{f}}(\omega=1-m)$ may be larger than ${ }_{\mathrm{s}} v_{\mathrm{f}}(\omega=0)$, i.e., ${ }_{\mathrm{B}} v_{\mathrm{f}}(\omega=0)<$ ${ }_{\mathrm{s}} v_{\mathrm{f}}(\omega=0)<{ }_{\mathrm{B}} v_{\mathrm{f}}(\omega=1-m)$; this leads to $0<f<1.21_{7}$ when $V^{*}=\sigma^{3} N$. Thus, the value of $f, 0.704$, obtained by eq 20 with $\beta=0.25$ appears more reasonable.

The theoretical $\gamma v s . V / \sigma^{3} N$ curves are illustrated in Figure 1. Solid lines were calculated

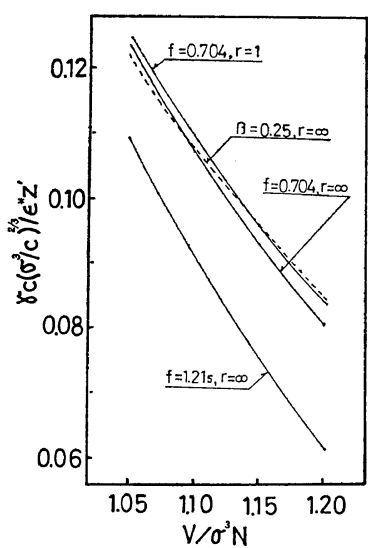

Figure 1. Calculated curves of reduced surface tension vs. reduced volume: cell volume model; ----, the changeable cell volume model.

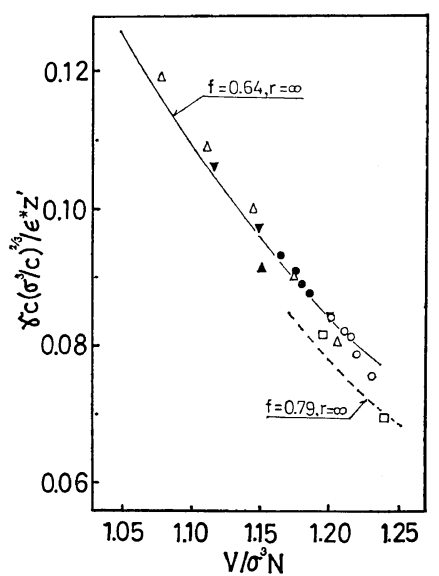

Figure 2. Reduced surface tension $v s$. reduced volume: - and ----, calcd; expt, $\triangle$, poly(isobutylene); ${ }^{3} \bigcirc$, poly (vinyl acetate) $;{ }^{4} \bullet$, polyethylene; ${ }^{4} \Delta$, polystyrene; ${ }^{4} \square, n-\mathrm{C}_{7} \mathrm{H}_{16},{ }^{10}$ and $\nabla$, $n-\mathrm{C}_{18} \mathrm{H}_{38}{ }^{10}$

by eq 21 with $(f=0.704, r=1),(f=704, r=\infty)$ and $\left(f=1.21_{7}, r=\infty\right), \quad V^{*}=\sigma^{3} N$ being assumed (constant cell volume $\operatorname{model}^{7}$ ), and a dashed line was calculated with $\beta=0.25$, and $r=\infty$, the cell volume being assumed to be allowed to change. Here the value of $\tilde{T}$ and $V^{*} / \sigma^{3} N$ corresponding to $V / \sigma^{3} N$ were determined by the $V / \sigma^{3} N$ vs. $\tilde{T}$ and $V^{*} / \sigma^{3} N$ vs. $\tilde{T}$ curves obtained previously. ${ }^{7}$ As shown in Figure 1, the constant cell volume model where $V^{*}=\sigma^{3} N$ does not 
A Hole Theory of Polymer Liquids and Glasses. V.

give a significantly different result from that of the changeable cell volume model. Accordingly we will use the former model for simplicity in the following discussion.

When $c=1, z=z^{\prime}, \beta=0.25$, and $V^{*}$ is taken to be the volume of solid state at the melting point, which is nearly equal to $\sigma^{3} N$, eq 14 is reduced to the final result for surface tension in the significant structure theory proposed by Ree, et al. ${ }^{9}$ for low-molecular-weight substances.

In Figure 2, experimental results are compared with those calculated for $n$-hydrocarbons and some polymers, where the molecular parameters obtained in previous paper $^{7}$ are used. It can be seen from the figure that by taking the term $\gamma\left(\sigma^{3} / c\right)^{2 / 3} / \varepsilon^{*} z^{\prime}$ for reduced surface tension, the principle of corresponding states for surface tension is fairly well satisfied. This seems to support the above-mentioned method for estimating surface area, and shows that the theory gives a reasonable $\tilde{\gamma} v s . V / \sigma^{3} N$ relation. Looking at details, however, we notice that $\tilde{\gamma}$ for $n$ hydrocarbons of lower carbon number is slightly lower than that for polymers. This tendency is clearly found in Figure 3 in which the surface tension of $n$-hydrocarbon at $20^{\circ} \mathrm{C}$ is plotted against the reciprocal of the carbon number. The data used are tabulated in Table I, where the molecular parameters are those obtained in the preceding paper. ${ }^{7}$ It is shown in Figure 3 that as the chain becomes longer the calculated curve of $\gamma$ deviates downwards from the experimental values. This tendency is also seen in

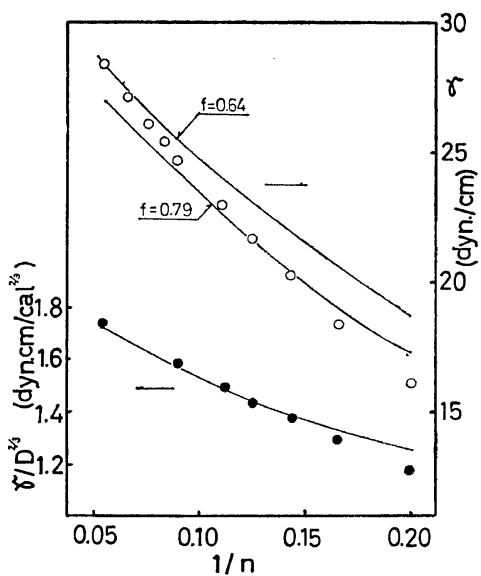

Figure 3. Surface tension of $n$-hydrocarbons at $20^{\circ} \mathrm{C}$ and its relation to cohesive energy density: $n$, the carbon number; - , calcd; $\bigcirc$, and $\bigcirc$ expt. ${ }^{10}$ The data used are tabulated in Table I.

the fact that the suitable value of $f$ is 0.64 for polymers, whereas it is 0.79 for $n$-hydrocarbons. One of the possible causes is the assumption by which the contribution from the chain configuration at the surface is neglected, i.e., from the term $J(T) \sum_{\lambda} g_{\lambda}$, which we will discuss in in little more detail later. Generally speaking, however, it can be said that the principle of corresponding states is approximately satisfied for the reduced surface tension $\gamma\left(\sigma^{3} / c\right)^{2 / 3} c / \varepsilon^{*} z^{\prime}$, as the theory suggests.

The molecular weight dependence of thermodynamic properties such as cohesive energy

Table I. Cohesive energy density and surface tension of $n$-hydrocarbons at $20^{\circ} \mathrm{C}$ with their molecular parameters ${ }^{a}$

\begin{tabular}{|c|c|c|c|c|c|c|c|c|c|c|}
\hline \multirow{2}{*}{$\begin{array}{l}\text { Carbon } \\
\text { number }\end{array}$} & \multirow{2}{*}{$V / \sigma^{3} N$} & \multirow{2}{*}{$\widetilde{T} \times 10^{2}$} & \multirow{2}{*}{$\begin{array}{c}\sigma^{3} N / c \\
\mathrm{cc} / \mathrm{mol}\end{array}$} & \multicolumn{2}{|c|}{$\begin{array}{c}D, \\
\mathrm{cal} / \mathrm{cc}\end{array}$} & \multicolumn{2}{|c|}{$\begin{array}{c}\gamma, \\
\text { dyn/cm }\end{array}$} & \multicolumn{2}{|c|}{$\begin{array}{c}\gamma / D^{2 / 3} \\
\operatorname{dyn~} \mathrm{cm} / \mathrm{cal}^{2 / 3}\end{array}$} & \multirow{2}{*}{$\widetilde{T}_{\mathrm{b}} \times 10^{2}$} \\
\hline & & & & $\operatorname{Expt}^{b}$ & Calcd & $\operatorname{Expt}^{c}$ & Calcd & Expt & Calcd & \\
\hline 5 & 1.254 & 6.61 & 72.5 & 50.2 & 51.8 & 16.1 & 17.3 & 1.182 & 1.252 & 6.98 \\
\hline 6 & 1.218 & 6.23 & 77.4 & 53.8 & 54.7 & 18.4 & 19.15 & 1.293 & 1.327 & 7.28 \\
\hline 7 & 1.196 & 5.92 & 81.7 & 56.1 & 56.7 & 20.3 & $20.5_{5}$ & 1.380 & 1.402 & 7.51 \\
\hline 8 & 1.179 & 5.68 & 85.5 & 57.9 & $(57.9)$ & 21.7 & 21.7 & 1.446 & 1.446 & 7.71 \\
\hline 9 & 1.166 & 5.50 & 88.5 & 59.5 & 59.4 & 22.9 & 22.7 & 1.506 & 1.501 & 7.95 \\
\hline 11 & 1.150 & 5.23 & 93.5 & 61.5 & 60.8 & 24.8 & 24.1 & 1.59 & 1.567 & 8.35 \\
\hline 18 & 1.117 & 4.72 & 103.4 & $(65.5)$ & 64.0 & 28.4 & 27.0 & 1.74 & 1.738 & 9.47 \\
\hline
\end{tabular}

a In calculation of $z^{\prime}$, the value of $r$ is taken to be equal to the carbon number. The calculated values are obtained by eq 21 and 24 with $f=0.79$.

b ref 12 . $\quad$ ref 10 . 


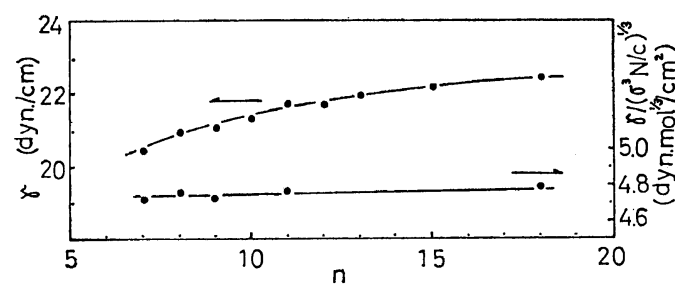

Figure 4. Carbon number dependences of surface tension $\gamma$ and $\gamma /\left(\sigma^{3} N / c\right)^{1 / 3}$ of $n$-hydrocarbons at the same reduced volume $V / \sigma^{3} N=1.19$.

density and internal pressure at the same temperature, is mainly attributable to higher density or lower values of $\tilde{V}$ of higher molecular weight substances, as shown previously. ${ }^{7}$ In the case of surface tension, however, the volume $v^{*} / c$ of the freely movable segment may also play an important role. For example, the surface tension $\gamma$ in $n$-hydrocarbons of different carbon numbers at the same reduced volume slightly increases as the carbon number increases, whereas $\gamma /\left(v^{*} / c\right)^{1 / 3}$ is almost independent of the carbon number as the theory predicts. This is illustrated in Figure 4. The data used are tabulated in Table I.

Temperature Dependence at Atmospheric Pressure

The temperature dependence of $\gamma$ is expressed by eq 22 or 23 in the present theory. On the other hand, if surface tension is expressed by the parachor $P$, i.e., $\gamma=(P / V)^{4}$ when the density of vapor is disregarded, we have

$$
-(\partial \ln \gamma / \partial T)_{p} / \alpha=4
$$

and

$$
-(\partial \ln \gamma / \partial T)_{p}=4(\partial \ln \tilde{V} / \partial \ln \tilde{T})_{\tilde{p}}
$$

These equations (eq 22, 23, 28, and 29) show that if the principle of corresponding states for the $\tilde{V}-\tilde{T}$ relation holds the quantity $-(\partial \ln \gamma / \partial T)_{p} / \alpha$ or $-(\partial \ln \gamma / \partial \ln T)_{p}$ should be a universal function of $\tilde{V}$ or $\tilde{T}$, when the contribution of $s$, which is very small, is ignored. Comparison of the calculated and experimental results is shown in Figure 5. The values of $\tilde{T}$ and $(\partial \ln \tilde{V} / \partial \ln \tilde{T})_{\tilde{p}}$ corresponding to $\tilde{V}$ were determined by the experimental $\tilde{V}-\tilde{T}$ relation. (As previously mentioned, ${ }^{7}$ the $\tilde{V}$ vs. $\tilde{T}$ curves of $n$-hydrocarbons and those of polymers do not superpose with each other in the range of larger $\tilde{V}$ or $\tilde{T}$. Accordingly the different values of $\tilde{T}$ and $(\partial \ln \tilde{V} / \partial \ln \tilde{T})_{\tilde{p}}$ against the same $\tilde{V}$ are given. The calculated curves of eq 22, 33, and 29 for $n$-hydrocarbons are also different from those of polymers). The experimental values

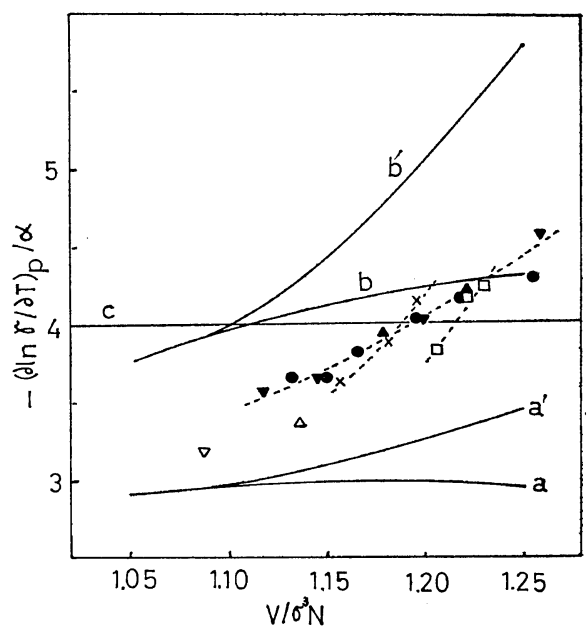

(a)

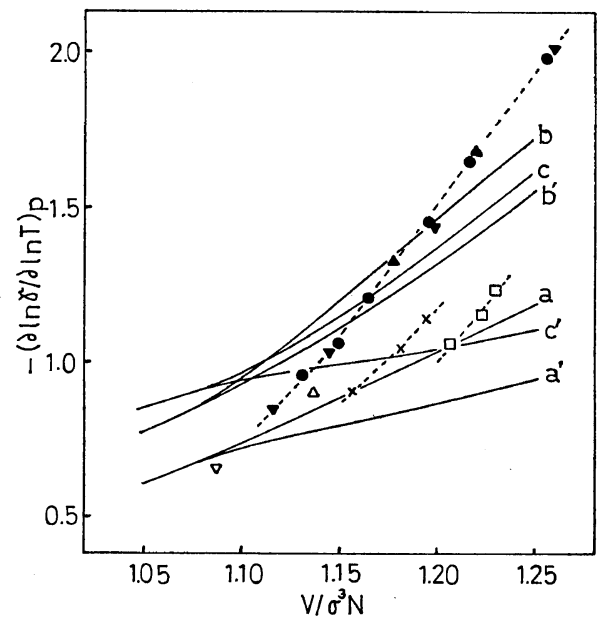

(b)

Figure 5. Temperature dependence of surface tension: expt, $\bigcirc, n$-hydrocarbons $(n=5 \sim 14) ;{ }^{10}$ A, $n-\mathrm{C}_{8} \mathrm{H}_{18} ;{ }^{10} \nabla, \quad n-\mathrm{C}_{18} \mathrm{H}_{38} ;{ }^{10} \times$, polyethylene; ${ }^{4} \triangle$, poly(dimethylsiloxane) ${ }^{3} \nabla$, poly(isobutylene), ${ }^{3}$ and $\square$, poly(vinyl acetate); 4 calcd, a, the present theory (eq 22 and 23) of $r=\infty$ and $f=0.64, \mathrm{~b}$, that of $r=\infty$ and $f=1.21_{5}$, and $\mathrm{c}$, parachor (eq 24 and 25) (prime, from the $\tilde{V}-\widetilde{T}$ relation for polymers, ${ }^{7}$ and no prime, from that for $n$-hydrocarbons ${ }^{7}$. 
A Hole Theory of Polymer Liquids and Glasses. V.

of $-(\partial \ln \gamma / \partial T)_{p} / \alpha$ (Figure $5 \mathrm{a}$ ) increase from about 3.5 for $V / \sigma^{3} N=1.10$ to about 4.5 for $V / \sigma^{3} N=1.25$ with increases in the reduced volume $V / \sigma^{3} N$, whereas according to eq $28 \mathrm{de}-$ rived from the parachor-equation this value is 4 . The present theory gives a reasonable magnitude of the temperature dependence of surface tension. The experimental results for $n$-hydrocarbons show the definitely universal relations $-(\partial \ln \gamma / \partial T)_{p} / \alpha v s . V / \sigma^{3} N$ and $-(\partial \ln \gamma / \partial \ln T)_{p} v s$. $V / \sigma^{3} N$, whereas these relations for polymers are not superposed on these universal curves and indicate somewhat different behavior, as shown in Figure 5. To show the nature of this difference, however, further investigations are necessary.

\section{Relation to Cohesive Energy Density}

Hildebrand and $\operatorname{Scott}^{11}$ obtained experimentally the relation $\gamma / V^{1 / 3} \propto D^{1.15}$ for low-molecularweight substances and proposed that the ratio of $\left(\gamma / V^{1 / 3}\right) / D$ would be constant for any substance, i.e., the so-called Hildebrand equation $\gamma / V^{1 / 3}=$ constant $\times D$.

In general, however, such ratios as $\left(\gamma / V^{1 / 3}\right) / D$ and $\left(\gamma / V^{1 / 3}\right) D^{1.15}$ should depend on temperature, because $\gamma$ is approximately proportional to $V^{-4}$, as shown in Figure 5 a, whereas $D$ is approximately proportional to $V^{-2} \cdot{ }^{12}$ In the present theory, the value of $\gamma /\left(D^{2} T\right)^{1 / 3}$ should depend almost wholly on the reduced temperature and the reduced volume as indicated by eq 27 . If we did not introduce the concept of the freely movable segment volume $v^{*} / c$ in the determination of surface area, the term $c^{1 / 3}$ in eq 25 would disappear and $\left(\gamma / V^{1 / 3}\right) / D$ would depend only on $\tilde{V}$ and $\tilde{T}$. The values of $D\left(25^{\circ} \mathrm{C}\right)$ and $\gamma\left(20^{\circ} \mathrm{C}\right)$ and the ratios of $D /\left(\gamma / V^{1 / 3}\right),\left(\gamma / V^{1 / 3}\right) / D^{1.15}$ and $r / D^{2 / 3}$ obtained from the data used by Hildebrand and $\mathrm{Scott}^{11}$ are tabulated in Table II. (Though the values of $\gamma, D$, and $V$ at different temperatures $20^{\circ} \mathrm{C}$ and $25^{\circ} \mathrm{C}$ are used, the various ratios obtained from these values may be regarded as those at the same temperature, about $20^{\circ} \mathrm{C}$, because the temperature dependence of $D V^{1 / 3}$, or $D^{1.15} V^{1 / 3}$, or $D^{2 / 3}$ and the temperature difference are both small). In Figure 6 , the ratio $\gamma / D^{2 / 3}$ listed in Table II is plotted against the boiling point temperature $T_{\mathrm{b}}$, because the boiling point may be a corresponding state at least for the same kind of molecules, i.e., the substance having higher $T_{\mathrm{b}}$ may show lower $\tilde{T}$ at the same temperature.

Table II. Surface tension and cohesive energy density of low-molecular-weight liquids

\begin{tabular}{|c|c|c|c|c|c|c|c|c|}
\hline & Substance & $\begin{array}{c}V^{\mathrm{a}} \\
\text { at } 25^{\circ} \mathrm{C}, \\
\mathrm{cc} / \mathrm{mol}\end{array}$ & $\begin{array}{c}D^{\mathrm{a}} \\
\text { at } 25^{\circ} \mathrm{C} \\
\mathrm{cal} / \mathrm{cc}\end{array}$ & $\begin{array}{c}\stackrel{\gamma}{\gamma}^{a} \\
\text { at } 20^{\circ} \mathrm{C} \\
\text { dyn } / \mathrm{cm}\end{array}$ & $\begin{array}{c}T_{\mathrm{b}^{\mathrm{b}}}, \\
{ }^{\mathrm{K}}\end{array}$ & $\begin{array}{c}\frac{D}{\gamma / V^{1 / 3}}, \\
\mathrm{cal} / \\
\text { dyn cm }\end{array}$ & $\begin{array}{l}r / V^{1 / 3} \\
D^{1.15} \\
\times 10^{2}\end{array}$ & $\frac{\gamma}{\underset{\mathrm{dal}^{2 / 3}}{D^{2 / 3} \mathrm{~cm}}}$ \\
\hline 1. & iso-Pentane & 117 & 45.6 & 15.0 & 282.5 & 13.90 & 3.78 & 1.177 \\
\hline 2. & n-Pentane & 116 & 49.7 & 16.0 & 301 & 15.14 & 3.69 & 1.181 \\
\hline 3. & Hexane & 132 & 53.3 & 18.4 & 342 & 14.78 & 3.72 & 1.300 \\
\hline 4. & Ethyl ether & 105 & 56.3 & 17.0 & 307.6 & 15.62 & 3.49 & 1.167 \\
\hline 5. & $n$-Octane & 164 & 57.0 & 21.8 & 398.7 & 14.26 & 3.81 & 1.472 \\
\hline 6. & Cyclohexane & 109 & 67.2 & 25.3 & 353 & 12.68 & 4.21 & 1.532 \\
\hline 7. & Carbon tetrachloride & 97 & 73.9 & $26.9_{5}$ & 349.8 & 12.61 & 4.16 & 1.531 \\
\hline 8. & $m$-Xylene & 123 & 77.4 & 28.9 & 412.3 & 13.30 & 3.92 & 1.587 \\
\hline 9. & Toluene & 107 & 79.2 & 28.5 & 383.8 & 13.20 & 3.95 & 1.548 \\
\hline 10. & Benzene & 89 & 83.7 & 29.0 & 333.1 & 12.86 & 4.02 & 1.518 \\
\hline 11. & Chloroform & 81 & 86.5 & $(28.5)$ & 334.2 & 13.10 & 3.91 & 1.461 \\
\hline 12. & Chlorobenzene & 102 & 90.2 & 33.56 & 405.1 & 12.52 & 4.07 & 1.670 \\
\hline 13. & 1,2 Dichloroethane & 79 & 96.1 & 32.2 & 356.7 & 12.82 & 3.93 & 1.532 \\
\hline 14. & Carbondisulfide & 61 & 100 & 32.3 & 319.3 & 12.20 & 4.12 & 1.501 \\
\hline 15. & Pyridine & 81 & 114.5 & 38.0 & 385 & 13.04 & 3.77 & 1.617 \\
\hline 16. & Bromine & 51 & 110.2 & 41.5 & 331.8 & 9.86 & 5.07 & 1.812 \\
\hline 17. & Methyliodide & 63 & $(139.2)$ & 50.8 & 315.4 & 11.80 & 4.06 & 1.892 \\
\hline
\end{tabular}

a ref $11 . \quad$ b ref 13 . 


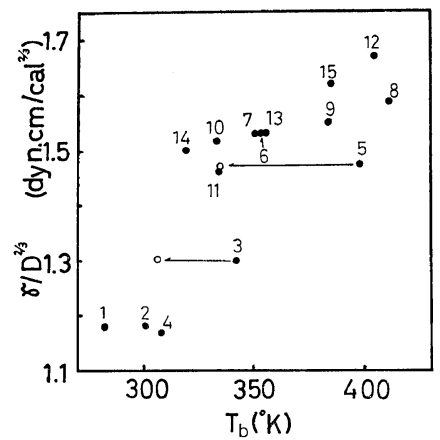

Figure 6. Relation between surface tension and cohesive energy density. The numbers indicate the substances in Table II.

As seen from Figure 6 the plot of $\gamma / D^{2 / 3}$ vs. $T_{\mathrm{b}}$ shows the existence of a correlation between them, whereas the other ratios such as $\left(\gamma / D^{1 / 3}\right) /$ $D^{1.15}$ and $D /\left(\gamma / V^{1 / 3}\right)$ do not show such a correlation against $T_{\mathrm{b}}$. This fact may support the concept of the freely movable segment at the surface. It is noticeable that the $\gamma / D^{2 / 3} v s . T_{\mathrm{b}}$ plot seems to be classified into two groups; those for chain-like $(1-5)$ and relatively spherical (6-15) molecules. This may be attributed to the fact that the boiling point is not exactly a corresponding state or, in other words, $\tilde{T}_{\mathrm{b}}\left(=T_{\mathrm{b}} / T^{*}\right)$ is not constant for all types of molecules, as indicated in the last columns of Tables I and III. The molecules having more intramolecular degrees of freedom seem to have a larger value of $\tilde{T}_{\mathrm{b}}$. This implies that not $\tilde{T}_{\mathrm{b}}\left(\equiv c k T_{\mathrm{b}} / \varepsilon^{*} z^{\prime}\right)$ but $k T_{\mathrm{b}} / \varepsilon^{*} z^{\prime}$ may be constant when a whole molecule is taken as a segment. This is suggested by Trouton's law. Therefore if a hypothetical boiling point of a chain-like molecule is chosen so as to have the same $\tilde{T}_{b}$ as that of a spherical one, it should be lower than the real boiling point. As an example we calculated such hypothetical boiling points for two chain-like molecules ( $n$-hexane and $n$ octane), using $\tilde{T}_{\mathrm{b}}=0.065$ for benzene and their characteristic temperatures $T^{*}$ listed in Table I. These points shown in Figure 6 appear to lie in line with the points of relatively spherical molecules also shown in the figure. Thus it can be seen that the $r / D^{2 / 3}$ vs. $\tilde{T}$ or $\tilde{V}$ plot may result in a single curve.

According to eq 27 , the value of $\gamma / D^{2 / 3}$ is calculated to be 1.366 for $\left(V / \sigma^{3} N=1.20, \tilde{T}=\right.$ $0.0613)$ and 1.820 for $\left(V / \sigma^{3} N=1.10, \tilde{T}=0.0430\right)$, when $T=293^{\circ} \mathrm{K}\left(20^{\circ} \mathrm{C}\right), z=z^{\prime}$ and $f=0.79$. (The term $s\left(=z^{\prime} / z\right)$ has no particular effect on this value. The value of $\tilde{T}$ against $\tilde{V}$ was again determined by the $\tilde{V}-\tilde{T}$ curve obtained previously $\left.{ }^{7}\right)$. The calculated values agree closely with the experimental ones shown in Figure 6 and Table II. This close agreement is also found for $n$-hydrocarbons, as shown in Figure 3 and Table I.

It has now been seen that $\gamma /\left(D^{2} T\right)^{1 / 3}$ depends almost entirely on the reduced state, as eq 27 predicts. The above arguments may also be valid for polymer liquids, as is suggested by the agreement of the theoretical calculation with experimental ones in $\gamma$ of polymer liquids. Though there is no direct way to measure cohesive energy density for polymer liquids, internal pressure $P_{\mathrm{i}}$ may be useful to estimate $D$ so far as the relation $P_{\mathrm{i}} / D \cong 1$ holds in the liquid state. ${ }^{7,12}$ In this case, exactly the same relation as eq 27 may also hold for the relationship between internal pressure and surface tension.

\section{Parachor}

Recently some investigators ${ }^{4,14}$ showed that the parachor concept is also applicable to surface tension of molten polymers and McGowan ${ }^{15}$ applied it to compressibility. We will now express the well-known molecular parameter, parachor, in terms of the molecular parameters involved in the present theory to give it a pnysical meaning. Parachor $\boldsymbol{P}$ is defined, if the density of vapor is ignored, as

$$
P=V \gamma^{1 / 4}
$$

Then, using eq 21 and 30 , we have

$$
P=0.839 \sigma^{3} N\left(\sigma^{3} N / c\right)^{1 / 12}\left(0.699 \varepsilon^{*} z^{\prime} / \sigma^{3}\right)^{1 / 4} F
$$

where

$$
F=\tilde{V}^{3 / 4}\left\{s(\tilde{V}-1+s)^{-1}\left(0.229-1.31_{2} f \tilde{T}\right)\right\}^{1 / 4}
$$

Here, $\boldsymbol{P}, \quad \sigma^{3} N$ and $\varepsilon^{*} z^{\prime} / \sigma^{3}$ are expressed in $\mathrm{cc} \mathrm{dyne}^{1 / 4} / \mathrm{cm}^{1 / 4}, \mathrm{cc} / \mathrm{mol}$ and $\mathrm{cal} / \mathrm{cc}$, respectively. Since $\gamma$ given by eq 21 is approximately proportional to $V^{-4}$, as shown in Figure 5 a, the function $F$ should be almost constant. In fact the value of $F$ calculated by eq 32 with the value of $f$ obtained before, i.e., $f=0.704$, is 
A Hole Theory of Polymer Liquids and Glasses. V.

$0.694 \pm(1.6 \%)$ in the range of $\tilde{V}=1.05-1.20$, no hole $\left(V=V^{*}=\sigma^{3} N\right)$. The condition $c^{1 / 12} \cong 1$ and may be regarded as a constant. As a result, may be satisfied only if we take an atomic when $c^{1 / 12}$ can be approximated to unity, eq 31 group or a molecule whose external degrees of with $F=0.694$ reduces to

$$
P=0.582\left(\sigma^{3} N\right)^{13 / 12}\left(0.699 \varepsilon^{*} z^{\prime} / \sigma^{3}\right)^{1 / 4}
$$

The quantity $\sigma^{3} N$ is a characteristic molar volume or an occupied molar volume, and $0.699 \varepsilon^{*} z^{\prime} / \sigma^{3}$ is the characteristic cohesive energy density or $D$ of the hypothetical liquid having

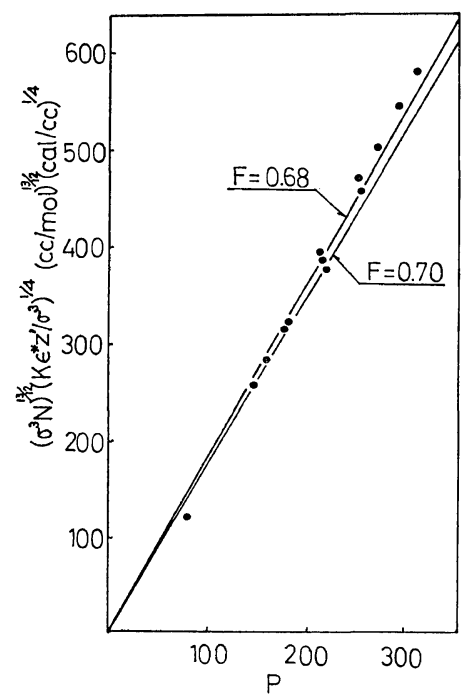

Figure 7. Relation between parachor and molecular parameters. The data used are tabulated in Table III. freedom do not essentially vary from three. Therefore, in the case of polymers, we can usually take a repeating unit.

As may be seen from the definition of eq 30 , the parachor is the molar volume at which surface tension is unity, and is an additive quantity as is well known. According to eq 31 or 33 , the value of the parachor is determined primarily by occupied volume and secondarily by cohesive energy density, whereas the parameter $c$ determined by the structure of a molecule is less effective insofar as an appropriate atomic group or molecule is taken. Since the former two quantities are almost additive, the parachor expressed by eq 31 may also be approximately additive. In Figure 7, the plot of $P$ vs. $\left(\sigma^{3} N\right)^{13 / 12}\left(0.699 \varepsilon^{*} z^{\prime} / \sigma^{3}\right)^{1 / 4}$ is shown. The former value, parachor, is calculated from the values given in the literature ${ }^{16}$ by the use of the additivity, and the latter is calculated from the molecular parameters obtained by the method described previously. ${ }^{7}$ These values are tabulated in Table III. The plot agrees closely with the theoretical prediction indicated by the solid lines.

\section{On The Present Model}

The most remarkable assumption in the present theory is that no difference is assumed in cell volume, hole fraction, and chain conforma-

Table III. Various molecular parameters

\begin{tabular}{|c|c|c|c|c|c|c|}
\hline Substance & $\begin{array}{c}\sigma^{3} N \\
\mathrm{cc} / \mathrm{mol}\end{array}$ & $\frac{0.699 \varepsilon^{*} z^{\prime}}{\begin{array}{c}\sigma^{3} \\
\mathrm{cal} / \mathrm{cc}\end{array}}$ & $T^{*} \times{ }^{\circ} \mathrm{K}$ & $\begin{array}{c}\left(\sigma^{3} N\right)^{13 / 12} \\
\times\left(0.699 \varepsilon^{*} z^{\prime} / \sigma^{3}\right)^{1 / 4}\end{array}$ & $P$ & $\tilde{T}_{\mathrm{b}} \times 10^{2}$ \\
\hline Benzene & 76.5 & 164 & 5.47 & 394 & 216.1 & 6.45 \\
\hline Toluene & 91.7 & 150 & 5.69 & 469 & 255.7 & 6.75 \\
\hline Ethylbenzene & 107 & 103 & 5.80 & 502 & 277.3 & 7.05 \\
\hline iso-Propylbenzene & 122.4 & 97.3 & 5.84 & 578 & 316.9 & - \\
\hline Dichloromethane & 52.7 & 147.2 & 4.70 & 256 & 147.2 & 6.66 \\
\hline Trichloromethane & 67.4 & 126.3 & 4.9 & 322 & 184.8 & 6.81 \\
\hline Carbon tetrachloride & 81.3 & 105.1 & 4.97 & 375 & 222.4 & 7.02 \\
\hline Dimethyl ketone & 59.0 & 133 & 4.61 & 282 & 162.0 & 7.15 \\
\hline Methyl acetate & 63.7 & $(147)$ & 4.31 & 314 & 178.8 & 7.66 \\
\hline Ethyl acetate & 78.9 & 130.4 & 4.5 & 385 & 218.4 & 7.77 \\
\hline n-Propyl acetate & 94.3 & 121 & 4.68 & 455 & 258.0 & 8.00 \\
\hline n-Butyl acetate & 113.4 & 114.4 & 4.78 & 545 & 297.6 & 8.39 \\
\hline$-\mathrm{CH}_{2} \cdot \mathrm{CH}_{2}-$ & 30.6 & 80.7 & - & 122 & 79.2 & - \\
\hline
\end{tabular}


tion between surface and bulk phases. Actually this may not be true. For example, there must be a segment density gradient in the surface layer and the conformation of polymer chains at the surface must differ from that in bulk. For dilute polymer solutions or an isolated polymer chain, the conformation of chain molecules at the surface has been theoretically studied by some investigators, ${ }^{17}$ but in the case of molten polymers no attempt seems to have been made. If we wish to solve this problem precisely, we may have to take another approach or another description of polymer liquids different from the present treatment. However we may conveniently express the effect of chain configuration in the present theory by assuming that the term $J(T) \sum_{\lambda} g_{\lambda}$ can be written in the form of $\left(j_{\mathrm{B}} g_{\mathrm{B}}\right)^{N_{\mathrm{B}}}\left(j_{\mathrm{S}} g_{\mathrm{S}}\right)^{N_{\mathrm{S}}}$ where $j_{\mathrm{B}}, j_{\mathrm{S}}, g_{\mathrm{B}}$, and $g_{\mathrm{S}}$ are independent of $N_{\mathrm{B}}$ and $N_{\mathrm{S}}$. Then, it leads to a correction term $-\varepsilon^{*} z^{\prime} c^{-1}\left\{\eta\left(v^{*} / c\right)^{2 / 3} c\right\}^{-1} T$ $\times \ln \left(j_{\mathrm{S}} g_{\mathrm{S}} / j_{\mathrm{B}} g_{\mathrm{B}}\right)$ to be added to the expression of surface tension of eq 14 . Therefore, this correction term can be included into the parameter $f$, i.e., $f$ in eq 14 may be replaced by the corrected $f, f_{\mathrm{c}}$, which is written

$$
f_{\mathrm{c}}=f+c^{-1} \tilde{V}(\tilde{V}-1+s) / s \ln \left(j_{\mathrm{s}} g_{\mathrm{s}} / j_{\mathrm{B}} g_{\mathrm{B}}\right)
$$

The parameter $f$ used in the foregoing argument may be regarded as that involving both terms in $\ln { }_{\mathrm{s}} v_{\mathrm{fo}} /{ }_{\mathrm{B}} v_{\text {fo }}$ (eq 15) and $\ln \left({ }_{\mathrm{s}} g_{\mathrm{s}} / j_{\mathrm{B}} g_{\mathrm{B}}\right)$. Since it can be considered that the number of chain configurations in the surface layer may be reduced as compared with that in bulk, it may be expected that $j_{\mathrm{S}} g_{\mathrm{S}}<j_{\mathrm{B}} g_{\mathrm{B}}$, and accordingly $f_{\mathrm{c}}<f$. This result seems to be consistent with the previously mentioned fact that the suitable value of $f$ for polymer liquids is somewhat smaller than that for low carbon number $n$ hydrocarbons. At any rate, however, we cannot argue on this point because of the simplicity of the theory. We can only say that the results obtained in this paper suggest that the chain connectivity does not have a significant effect on the difference in the configurational entropy between surface and bulk phases in the temperature range considered here, other than the contribution through the parameter $c$, or $\tilde{T}$.

Acknowledgment. The author wishes to thank Prof. T. Hata of the Tokyo Institute of Tech- nology for his continuing interest and helpful discussions.

\section{LIST OF SYMBOLS}

$a$ distance between the nearest neighbor sites

B, as subscript designates the bulk phase

$c$, one third of external degrees of freedom per segment

$D$, cohesive energy density

$d$, diameter of an equivalent sphere in the evaluation of surface area

$F, \quad$ function defined by eq 32

$f$, function defined by eq 15

$f_{\mathrm{c}}$, corrected function of $f$

$g$, number of configurations of molecules arranged on the lattice per segment

$\sum_{\lambda} g_{\lambda}$, total number of configurations of molecules arranged on the lattice

$J(T)$, partition function for intrasegmental degrees of freedom as a function of temperature

$j$, partition function per segment for intrasegmental degrees of freedom

$K, \quad$ function defined by eq 16

$k, \quad$ Boltzmann's constant

$M, \quad$ total number of lattice sites

$m$, ratio of the number of the nearest neighbor sites in the surface layer to that in the interior of bulk

$N, \quad$ total number segments

$n_{\mathrm{ol}}^{*}$, most probable number of segmenthole pairs

$\boldsymbol{P}, \quad$ parachor (eq 30)

$q$, kinetic part of partition functions per segment for external degree of freedom

$R, \quad$ gas constant

$r$ number of segments of a polymer chain

S, as subscript designates the surface phase

$s$, ratio of coordination number of a segment to that of a lattice site, $z^{\prime} / z$

$T$, absolute temperature

$\tilde{T}, \quad$ reduced temperature defined as $c k T / \varepsilon^{*} z^{\prime}$

$T^{*}$. characteristic temperature defined as $\varepsilon^{*} z^{\prime} / c k$

$T_{\mathrm{b}}$, boiling point temperature

$V$, volume 
A Hole Theory of Polymer Liquids and Glasses. V.

$\tilde{V}, \quad$ reduced volume defined by $\tilde{V}=V / V^{*}=$ $M / N$

$V^{*} \quad$ volume of cells occupied with segments, $v^{*} N$

$v^{*}, \quad$ volume of a cell

$v_{\mathrm{f}}$, free volume defined by $\int_{\text {cell }}$ $\times \exp \{-E(\rho)+E(0)\} / k T \mathrm{~d} \rho, \quad$ where $E(\rho)$ is the average potential energy of a segment as a function of the distance $\rho$ from the center of the cell $\bar{v}_{\mathrm{f}}$, average free volume, i.e., $v_{\mathrm{f}}$ averaged over the admissible combinations for mixing of molecules and holes on the lattice

$v_{\text {fo, }}$ free volume when all the nearest neighbor sites are occupied with segments $(\omega=0)$, (eq 3 )

$v_{\mathrm{f} 1}$, free volume when all the nearest neighbor sites are empty $(\omega=1)$, (eq 3 )

$Z$, partition function

$z$, coordination number of a lattice site

$z^{\prime}$, coordination number of a segment

$\alpha, \quad$ thermal expansion coefficient

$\beta$, parameter with which the width of square well potential for the perpendicular motion toward the outside at surface is expressed as $a-\sigma(1-\beta)$

$\gamma, \quad$ surface tension

$\tilde{\gamma}, \quad$ reduced surface tension, $\gamma\left(\sigma^{3} / c\right)^{2 / 3} c / \varepsilon^{*} z^{\prime}$

$\varepsilon^{*}$, potential energy at minimum of potential curve of Lennard-Jones 12-6 potential

$\eta, \quad$ shape factor in eq 13

$\sigma, \quad$ distance between segment centers at which potential energy is zero in Lennard-Jones $12-6$ potential

$\varphi(\rho)$, mean potential energy per segmentsegment pair when the segment deviates from the center of the cell by the distance $\rho$

$\phi$, quantity defined by eq 8 , which is equal to $\omega_{\mathrm{B}}{ }^{*}\left(=\omega_{\mathrm{S}}{ }^{*}\right)$

$\Omega, \quad$ surface area

$\omega$, fraction of empty sites in the nearest neighbors $\omega^{*}, \quad$ most probable value of $\omega$

\section{REFERENCES}

1. H. Schonhorn, J. Chem. Phys., 43, 2041 (1965); H. Schonhorn and L. H. Scharp, J. Polym. Sci., Part A, 3, 569 (1965); L. H. Scharp, F. W. Ryan, and H. Schonhorn, J. Polym. Sci., Part A-2, 4, 538 (1966).

2. R. H. Dettre and R. E. Johnson Jr., J. Colloid Interfac. Sci., 21, 365 (1966).

3. R. J. Roe, Proc. Nat. Acad. Sci. U. S., 56, 819 (1966).

4. T. Hata, Hyomen (Surface), 6, 281 (1968); S. Kitagawa and T. Hata, Preprint, The 4-th Symposium on Adhesion and Adhesive in Japan, Osaka, June 9, 1966, p 35; Y. Sasaki and T. Hata, Preprint, SPSJ 16th Annual Meeting, Tokyo, May 27, 1967, III I 27 p 400.

5. I. Prigogine and L. Saraga, J. Chem. Phys., 49, 399 (1952).

6. V. S. Nanda and R. Simha, J. Phys. Chem., 68, 3158 (1964).

7. T. Nose, Polymer J., 2, 124 (1971).

8. e.g., J. A. Barker, "Lattice Theory of the Liquid State," Pergamon Press, Oxford, 1963.

9. T. S. Ree and H. Eyring, J. Chem. Phys., 41, 524 (1964).

10. J. J. Jasper and E. V. Kring, J. Amer. Chem. Soc., 77, 1019 (1955).

11. J. H. Hildebrand and K. L. Scott, "The Solubility of Nonelectrolites," 3 rd ed, Reinhold, New York, N. Y., 1950, Chapter XXI.

12. G. Allen, G. Gee, and G. J. Wilson, Polymer, 1, 456 (1960).

13. J.H. Perry, "Chemical Engineering Handbook," McGraw-Hill, New York, N. Y., 1950.

14. R. J. Roe, J. Phys. Chem., 69, 2809 (1965).

15. J. C. McGowan, Polymer, 8, 57 (1967); 10, 841 (1969).

16. S. Glasstone, "The Elements of Physical Chemistry," Maruzen Company Ltd., Tokyo, 1954.

17. e.g., R. Simha, H. L. Frisch, and F. R. Eirich, J. Phys. Chem., 57, 584 (1953); W. C. Forsman and R. E. Hughes, J. Chem. Phys., 38, 2118, 2123, 2130 (1963); A. Silberberg, J. Phys. Chem., 66, 1872, 1884 (1962); J. Chem. Phys., 46, 1105 (1967); E. A. DiMarzio, ibid., 42, 2101 (1965); R. J. Roe, ibid., 43, 1591 (1965). 